\title{
Dehydrogenation Adjacent to Carbonyls Using Palladium-Allyl Intermediates
}

\author{
Aneta Turlik \\ Yifeng Chen \\ Timothy R. Newhouse* \\ Synlett 2016, 27, 331.
}

References 6, 21, and 24 were updated for the final online and print versions. We apologize for the production error. 